\title{
Comparação entre Nanocompósitos de Polietileno/ Nanotubos de Carbono e Polietileno/Nanolâminas de Grafeno Obtidos por Polimerização In Situ
}

\author{
Fabiana de Carvalho Fim, Gislaine Radaelli, Denise Schermann Azambuja, Griselda Barrera Galland \\ Instituto de Química, Universidade Federal do Rio Grande do Sul - UFRGS
}

\begin{abstract}
Resumo: Nanocompósitos de polietileno/nanotubos de carbono foram sintetizados através da polimerização in situ para serem comparados com nanocompósitos de polietileno/nanolâminas de grafeno, obtidos nas mesmas condições. Os nanocompósitos de polietileno/NTC foram obtidos com boas atividades catalíticas e foram caracterizados por DSC e MET. Os nanocompósitos com NG apresentaram melhor estabilidade térmica que os de NTC, porem não houve diferenças significativas nas propriedades dinâmico-mecânicas. No estudo da condutividade elétrica os nanocompósitos $\mathrm{PE} / \mathrm{NTC}$ atingiram condutividades de materiais semicondutores com menor teor de nanocarga que os de PE/NG.
\end{abstract}

Palavras-chave: Nanolâminas de grafeno, nanotubos de carbono, polietileno, nanocompósitos.

\section{Comparison between Polyethylene/Carbon Nanotubes and Polyethylene/Graphene Nanosheets Nanocomposites Obtained by In Situ Polymerization}

\begin{abstract}
Polyethylene/carbon nanotubes, PE/NTC, nanocomposites were synthesized by in situ polymerization for comparison with polyethylene/graphene nanosheets, $\mathrm{PE} / \mathrm{NG}$, nanocomposites obtained in the same conditions. The nanocomposites of polyethylene/NTC were obtained with good catalytic activities and were characterized by DSC and TEM. The nanocomposites with NG showed better thermal stability than with NTC, however, no significant differences in dynamic mechanical properties were found. In the electrical conductivity study, PE/NTC nanocomposites reached conductivities of semiconductor materials at lower content of filler than PE/NG nanocomposites.
\end{abstract}

Keywords: Graphene nanosheets, carbon nanotubes, polyethylene, nanocomposites.

\section{Introdução}

A fabricação de nanocompósitos poliméricos é importante para a geração de novos materiais com alto desempenho e multifuncionalidades. A incorporação de cargas nanométricas em uma matriz polimérica combina a leveza, flexibilidade e transparência de polímeros com as propriedades das nanocargas e é amplamente utilizada nas indústrias automobilística, aeronáutica, aeroespacial e de embalagens.

Desde a sua descoberta em 1991, os nanotubos de carbono (NTCs) têm sido o foco de investigação considerável. Devido às suas notáveis propriedades mecânicas, elétricas e térmicas, sua alta flexibilidade, baixa densidade de massa e alta razão de aspecto, os NTCs transformaram-se em potenciais candidatos para a preparação de nanocompósitos poliméricos ${ }^{[1,2]}$.

Existem dois tipos básicos de NTC: nanotubos de carbono de parede única (NTCPU) e nanotubos de carbono de paredes múltiplas (NTCPM). A obtenção de um tipo ou outro está diretamente relacionada com os procedimentos de síntese. NTCPU pode ser considerado como uma única folha de grafeno enrolada em um cilindro sem costura. Uma lâmina de grafeno é formada por carbonos hibridizados $\mathrm{sp}^{2}$, que estão ligados covalentemente de modo hexagonal. NTCPM consistem em cilindros de grafeno aninhados coaxialmente em torno de um núcleo central oco com separação interlamelar de aproximadamente $0,34 \mathrm{~nm}$. Os átomos de carbono no cilindro têm caráter parcial $\mathrm{sp}^{3}$, que aumenta à medida que o raio de curvatura do cilindro diminui ${ }^{[3]}$.

Outra nanocarga que tem atraído muito a atenção dos pesquisadores é a grafite. Grafite é quimicamente similar aos nanotubos de carbono e estruturalmente análoga aos silicatos em camada ${ }^{[4]}$, sendo por isso uma nanocarga potencial para uso em materiais poliméricos. O Brasil é o terceiro produtor mundial de grafite, precedido pela China e Índia, possuindo grandes reservas naturais ${ }^{[5]}$. A grafite, que é um material de baixo custo (commodity), é um mineral cinza metálico encontrado na natureza na forma de flocos ou em pó com vários tamanhos de partícula ${ }^{[6]}$. Cada camada que compõe a grafite é formada por lâminas de grafeno. A estrutura especial da grafite a torna interessante para várias aplicações devido a sua ótima condutividade elétrica e térmica, excelentes propriedades lubrificantes e resistência a altas temperaturas. A condutividade elétrica e térmica da grafite, assim como a dos NTC, se deve às ligações $\pi$ deslocalizadas, resultado da hibridização $\mathrm{sp}^{2}$, e a maciez e a ação lubrificante se devem às fracas ligações entre as lâminas de grafeno, que facilitam o deslizamento dessas lâminas umas sobre as outras ${ }^{[6]}$. Recentemente, o interesse industrial na grafite tem crescido muito, pois a partir deste material é possível obter o grafeno. Foi

Autor para correspondência: Griselda Barrera Galland, Instituto de Química, Universidade Federal do Rio Grande do Sul - UFRGS, Av. Bento Gonçalves, 9500, CEP 91501-970, Porto Alegre, RS, Brasil, e-mail: griselda.barrera@ufrgs.br 
descoberto que o grafeno, como condutor de eletricidade, é similar ao cobre, como condutor de calor, supera todos os outros materiais conhecidos e é um dos materiais mais fortes e mais duros que existe, mas, apesar disso, pode ser esticado até $25 \%$ de seu comprimento ${ }^{[7]}$. A condutividade térmica de uma monolâmina de grafeno foi estudada por Balandin e colaboradore $\mathrm{s}^{[8]}$, que encontraram o valor de aproximadamente $5.000 \mathrm{~W} / \mathrm{mK}$, este valor é muito superior quando comparado com os nanotubos de carbono de parede simples $(2.980 \mathrm{~W} / \mathrm{mK})$.

Outra grande vantagem deste material sobre os NTC é que o grafeno pode ser obtido a partir da grafite por meio de métodos de esfoliação, seja mecânico ou químico. O método de esfoliação mecânica é descrito pela descamação das lâminas de grafeno do floco de grafite com o uso de uma fita adesiva ${ }^{[7]}$. Já o método de esfoliação química consiste na modificação da estrutura da grafite por meio de tratamentos químicos e físicos ${ }^{[9-15]}$. Por este método é possível reduzir o número de lâminas de grafeno a dimensões nanométricas obtendo-se as nanolâminas de grafeno (NG).

A dispersão homogênea de NTCs e de NG em polímeros, para obter nanocompósitos, é relativamente difícil de alcançar pelo método clássico de fusão, especialmente em matrizes apolares tais como poliolefinas. Seu pequeno tamanho, grande área superficial e a presença de elétrons $\pi$ altamente deslocalizados em suas superfícies os tornam suscetíveis a forças de van der Waals que promovem a agregação, levando a muitos sítios de defeito nos compósitos formados ${ }^{[1,2,16]}$. Uma distribuição homogênea e boa aderência interfacial entre as nanocargas e a matriz polimérica são cruciais para a preparação bem sucedida de nanocompósitos. Portanto, a modificação não covalente na superfície dos NTCs, realizada recentemente pela polimerização in situ de olefinas, representa um método original e eficiente para formar NTCs revestidos de forma homogênea. A superfície dos nanotubos é previamente tratada com um sistema catalítico adequado e a polimerização do monômero inicia diretamente na superfície dos NTC, ocorrendo assim, a formação do polímero ao redor dos nanotubos. Este processo original, chamado de técnica de polimerização de enchimento (TPE) foi inicialmente investigado na polimerização in situ utilizando catalisadores Ziegler-Natta ${ }^{[17]}$. A partir disso, o método foi estendido para os catalisadores metalocênicos e consiste, primeiramente, em ancorar metilaluminoxano (MAO), o cocatalisador, na superfície dos $\mathrm{NTCs}^{[1,17-22]}$. Outros autores tem ligado diretamente o catalisador metalocênico diretamente ao suporte de NTCPMs, previamente oxidado, de maneira a ligar o catalisador aos grupos funcionais- $\mathrm{COOH}$ ou $-\mathrm{OH}$ criados nos NTCs durante a oxidação ${ }^{[23,24]}$.

A adição de monômero olefínico leva à síntese de cadeias poliméricas exclusivamente localizadas nos arredores da superfície do NTC que, com o aumento de massas moleculares, precipita-se sobre os nanotubos, cobrindo-os e levando a uma eficiente desaglomeração[1,17,22].

Nos últimos anos nosso grupo de pesquisa vem trabalhando na obtenção de nanolâminas de grafeno (NG) (empilhamento de poucos grafenos) através de esfoliação química e térmica da grafite e na preparação de nanocompósitos de polietileno $(\mathrm{PE})^{[25,26]}$ e de polipropileno $(\mathrm{PP})^{[27-29]}$ utilizando a técnica de polimerização in situ. Nosso primeiro trabalho nesta área ${ }^{[25]}$, foi citado em uma recente revisão ${ }^{[30]}$ como sendo o primeiro utilizando esta técnica para obter nanocompósitos de $\mathrm{PE} / \mathrm{NG}$.

O objetivo deste trabalho é de obter nanocompósitos de polietileno com NTCPM por polimerização in situ, nas mesmas condições experimentais com as que foram obtidos os nanocompósitos de polietileno com NG, e poder assim comparar a influência das duas nanocargas nas propriedades dos nanocompósitos.

\section{Parte Experimental}

\section{Materiais e métodos}

Todas as reações de manipulação foram realizadas sob atmosfera inerte (nitrogênio ou argônio) usando a técnica padrão Schlenk. Tolueno (Nuclear) foi destilado com sódio metálico e benzofenona. MAO (Witco, $5 \% \mathrm{p} / \mathrm{p} \mathrm{Al}$ em tolueno) e bis(ciclopentadienil)zircônioIV $\left(\mathrm{Cp}_{2} \mathrm{ZrCl}_{2}\right)$ (Sigma-Aldrich) foram usados como recebidos. Os nanotubos de carbono de parede múltipla (Baytubes C $150 \mathrm{P}$ - Bayer) sofreram tratamento descrito a seguir bem como a grafite expandida (Grafite Micrograf HC11 - Nacional de Grafite Ltda.).

\section{Tratamento dos NTCPMs e GN}

Os NTCPMs foram suspendidos em tolueno e tratados com $15 \%$ (p/p) de MAO. A suspensão ficou em agitação durante $30 \mathrm{~min}$.

A grafite expandida foi suspendida em etanol $70 \%$ e a suspensão foi tratada em ultrassom durante $8 \mathrm{~h}$ obtendo-se as nanolâminas de grafeno (NG). Após esse tratamento as NG foram suspendidas em tolueno e tratadas com $15 \%(\mathrm{p} / \mathrm{p})$ de MAO durante $30 \mathrm{~min}$. Mais detalhes sobre a síntese e caracterização das nanolâminas de grafeno e nanocompósitos $\mathrm{PE} / \mathrm{GN}$ podem ser encontrados nas referencias 25 e 26.

\section{Síntese dos nanocompósitos}

As polimerizações de etileno foram realizadas em um reator Parr com capacidade de $100 \mathrm{ml}$. As reações ocorreram a $70{ }^{\circ} \mathrm{C}$, durante $30 \mathrm{~min}$., sob agitação de $200 \mathrm{rpm}$ e 2,8 bar de pressão de etileno. Tolueno foi utilizado como solvente, MAO como cocatalisador $(\mathrm{Al} / \mathrm{Zr}=1000)$ e $\mathrm{Cp}_{2} \mathrm{ZrCl}_{2}$ como catalisador $(2 \mu \mathrm{mol})$. As nanopartículas, previamente tratadas com MAO, foram colocadas no reator como carga em quantidades variáveis $(0,1$ a $0,5 \mathrm{~g})$.

\section{Caracterização das nanocargas e dos nanocompósitos}

A temperatura de fusão e a cristalinidade dos nanocompósitos foram medidas em um calorímetro diferencial de varredura Perkin Elmer, modelo DSC-4 com uma taxa de aquecimento de $10^{\circ} \mathrm{C} \cdot \mathrm{min}^{-1}$ e uma faixa de temperatura de 30 a $160{ }^{\circ} \mathrm{C}$. $\mathrm{O}$ ciclo de aquecimento foi realizado duas vezes para eliminar a história térmica do material, mas apenas os resultados do segundo aquecimento foram considerados. $\mathrm{O}$ teor de cristalinidade 
$\left(\mathrm{X}_{\mathrm{c}}\right)$ dos polímeros foi calculado a partir do calor de fusão da amostra, $\Delta \mathrm{H}_{\mathrm{f}}$, (área da curva endotérmica) e o calor de fusão do polietileno completamente cristalino, $\Delta \mathrm{H}_{\mathrm{f}}^{\circ}$, $(64,5 \mathrm{cal} / \mathrm{g} \text { ou } 269,9 \mathrm{~J} / \mathrm{g})^{[31]}$.

As imagens das nanocargas de microscopia eletrônica de transmissão (MET) foram obtidas usando um microscópio JEOL 2010 operando a 200 kV. As amostras foram suspensas em acetona e posteriormente colocadas em um banho de ultrassom por $15 \mathrm{~min}$. Uma gota da suspensão foi depositada em uma grade de cobre de 300 mesh coberta com um filme de carbono amorfo.

As imagens dos nanocompósitos foram obtidas em um microscópio JEOL 1011 operando a $100 \mathrm{kV}$. As amostras foram suspensas em decalina a $160{ }^{\circ} \mathrm{C}$. Uma gota da suspensão foi depositada em uma grade de cobre de 300 mesh coberta com um filme de carbono amorfo.

A estabilidade térmica dos nanocompósitos foi determinada pela análise termogravimétrica (TGA) com um analisador Universal V2.6D (TA Instruments) a uma taxa de aquecimento de $20^{\circ} \mathrm{C} / \mathrm{min}$. As amostras (de 10 a $15 \mathrm{mg}$ ) foram aquecidas de $25 \mathrm{a} 600{ }^{\circ} \mathrm{C}$ usando atmosfera inerte (nitrogênio).

Os resultados dinâmico-mecânicos foram obtidos usando o analisador DMA (TA Instrument modelo Q800). As amostras foram analisadas no modo único cantiliver a uma frequência de $1 \mathrm{~Hz}$, a um nível de deformação de $0,1 \%$ no intervalo de temperatura de $-120{ }^{\circ} \mathrm{C}$ a $120{ }^{\circ} \mathrm{C}$. A taxa de aquecimento foi de $3{ }^{\circ} \mathrm{C} / \mathrm{min}$. O teste foi executado usando barras retangulares com dimensões de, aproximadamente, $17 \times 13 \times 3 \mathrm{~mm}$. Estas foram preparadas em uma mini-injetora Haake, modelo miniJet. A injeção foi a $165{ }^{\circ} \mathrm{C}$ com uma pressão de 250 bar durante 4 seg.

As medidas de impedância foram obtidas com filmes dos nanocompósitos colocados entre dois eletrodos de aço inoxidável montados em um suporte de resina epóxi. A espessura dos filmes variaram de 50 a $70 \mu \mathrm{m}$ para os nanocompósitos PE/NG e 10 a $20 \mu \mathrm{m}$ para os nanocompósitos PE/NTC e a área foi de $1,5 \mathrm{~cm}^{2}$ para ambos. Estes filmes foram preparados em um prensa Carver a $160{ }^{\circ} \mathrm{C}$, com uma força de 5 ton.cm durante 3 min. As medidas de impedância foram realizadas em um AUTOLAB PGSTAT 30/FRA 2 com uma faixa de frequência de $1 \mathrm{MHz}$ a $100 \mathrm{mHz}$ e a amplitude de voltagem sinusoidal foi $20 \mathrm{mV}$.

\section{Resultados e Discussão}

\section{Síntese e caracterização dos nanocompósitos PE/NTC}

O método de polimerização in situ para a produção de nanocompósitos poliméricos envolve a mistura da carga no monômero puro (ou monômeros múltiplos), ou uma solução de monômeros, seguida pela polimerização, na presença do sistema catalítico e da carga dispersa. Depois de decorrido o tempo de polimerização, o nanocompósito é precipitado em um solvente adequado, para posterior lavagem e secagem ${ }^{[30]}$. A cadeia polimérica pode se formar entre e ao redor da carga e dessa maneira promove naturalmente sua esfoliação. Por isso, a polimerização in situ tem sido usada para esfoliar e dispersar cargas em matrizes poliméricas com mais eficiência do que outros métodos, como fusão da matriz polimérica. Para testar a eficiência deste método os nanocompósitos obtidos foram caracterizados por diferentes técnicas e os resultados serão analisados a seguir.

A Tabela 1 mostra os percentuais de NTC nos nanocompósitos calculados a partir da quantidade de nanocarga colocada no reator e o rendimento final de nanocompósito. Esses percentuais variaram de 1,5 a $10,5 \%(\mathrm{p} / \mathrm{p})$ de NTC.

A atividade catalítica dos nanocompósitos foi um pouco menor do que do polietileno puro, mas não mostrou uma tendência consistente de queda com o aumento da quantidade de NTC. Não houve uma diferença significativa na atividade catalítica e isso indica que os NTC, quando tratados, não desativam o catalisador significativamente.

Os dados de $\mathrm{T}_{\mathrm{m}}$ (temperatura de fusão), $\mathrm{T}_{\mathrm{c}}$ (temperatura de cristalização) e cristalinidade das amostras foram determinados por Calorimetria Exploratória de Varredura (DSC) e estão listados na Tabela 2.

De acordo com a Tabela 2, percebe-se que a $\mathrm{T}_{\mathrm{m}}$ e a $\mathrm{T}_{\mathrm{c}}$ não foram significativamente influenciadas pela presença dos NTC. Por outro lado, o grau de cristalinidade $\left(\mathrm{X}_{\mathrm{c}}\right)$ teve uma variação, mas não houve uma tendência relacionada com o aumento da porcentagem de NTC. Estes resultados são similares aos obtidos com os nanocompósitos de $\mathrm{PE} / \mathrm{NG}^{[25]}$.

A morfologia dos NTC e dos nanocompósitos foi estudada por microscopia eletrônica de transmissão (MET). A Figura 1 mostra imagens representativas

Tabela 1. Nanocompósitos obtidos com diferentes percentuais de NTC e atividade catalítica.

\begin{tabular}{lcccc}
\hline $\begin{array}{c}\text { Nome da } \\
\text { amostra }\end{array}$ & $\begin{array}{c}\text { Peso do } \\
\text { polímero }(\mathbf{g})\end{array}$ & $\begin{array}{c}\text { Massa de } \\
\text { NTC (g) }\end{array}$ & $\begin{array}{c}\text { \% de } \\
\text { NTC (p/p) }\end{array}$ & $\begin{array}{c}\text { Atividade } \\
\text { Catalítica* }\end{array}$ \\
\hline PE puro & 8,1 & 0 & 0,0 & 2881 \\
PE-N1 & 6,8 & 0,1 & 1,5 & 2435 \\
PE-N2 & 5,3 & 0,12 & 2,2 & 1905 \\
PE-N3 & 5,3 & 0,25 & 4,7 & 1886 \\
PE-N4 & 5,0 & 0,25 & 5,0 & 1784 \\
PE-N5 & 7,1 & 0,5 & 7,0 & 2539 \\
PE-N6 & 5,9 & 0,5 & 8,4 & 2112 \\
PE-N7 & 5,5 & 0,5 & 9,1 & 1962 \\
PE-N8 & 4,8 & 0,5 & 10,5 & 1700 \\
\hline *(kgPol/molZr.h.br). & & &
\end{tabular}

$*$ (kgPol./molZr.h.bar).

Tabela 2. Resultados de temperatura de fusão $\left(\mathrm{T}_{\mathrm{m}}\right)$, temperatura de cristalização $\left(T_{c}\right)$ e cristalinidade $\left(X_{c}\right)$.

\begin{tabular}{lcccc}
\hline $\begin{array}{l}\text { Nome da } \\
\text { amostra }\end{array}$ & \% de NTC & $\mathbf{T}_{\mathbf{m}}\left({ }^{\circ} \mathbf{C}\right)$ & $\mathbf{T}_{\mathbf{c}}\left({ }^{\circ} \mathbf{C}\right)$ & $\mathbf{X}_{\mathbf{c}}(\boldsymbol{\%})$ \\
\hline PE puro & 0,0 & 130 & 120 & 66 \\
PE-N1 & 1,5 & 131 & 119 & 65 \\
PE-N2 & 2,2 & 130 & 119 & 74 \\
PE-N3 & 4,7 & 130 & 119 & 75 \\
PE-N4 & 5,0 & 130 & 120 & 75 \\
PE-N5 & 7,0 & 130 & 120 & 50 \\
PE-N6 & 8,4 & 130 & 119 & 44 \\
PE-N7 & 9,1 & 130 & 119 & 65 \\
PE-N8 & 10,5 & 131 & 119 & 68 \\
\hline
\end{tabular}




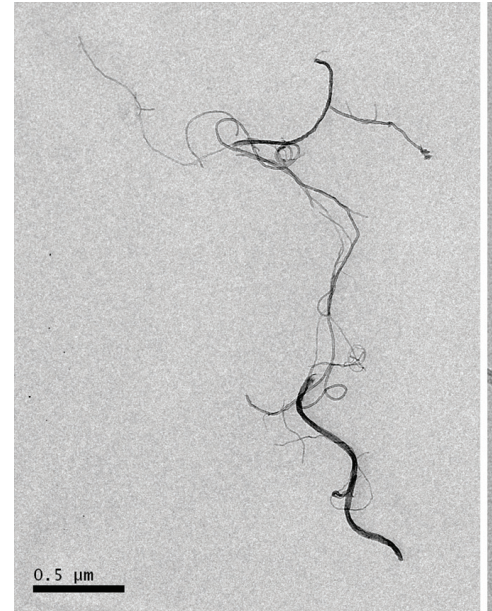

(a)

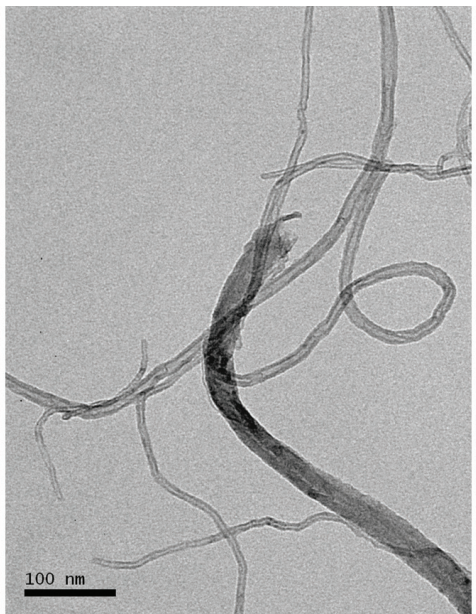

(b)

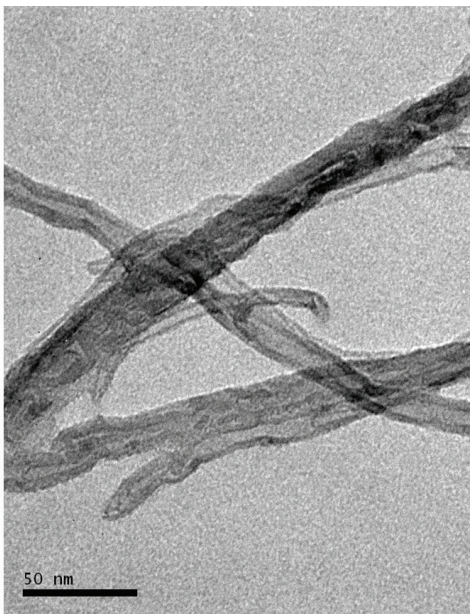

(c)

Figura 1. Micrografias de MET dos NTC puros.

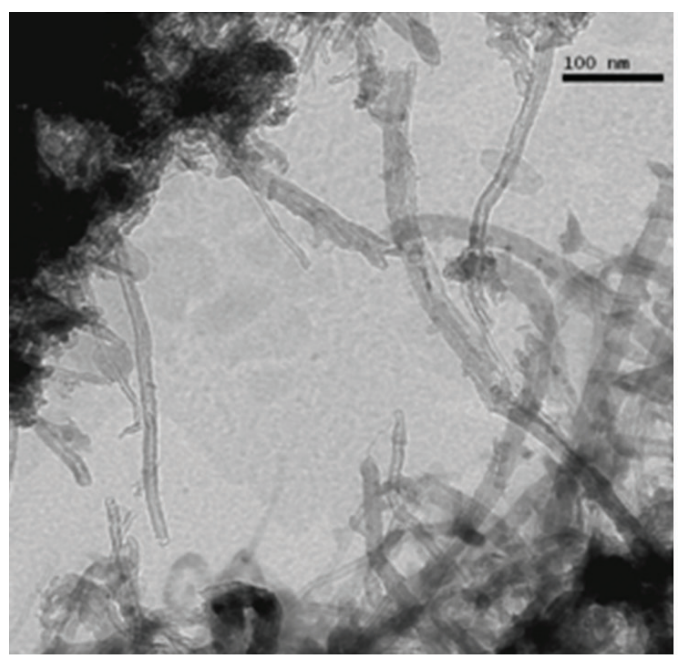

(a)

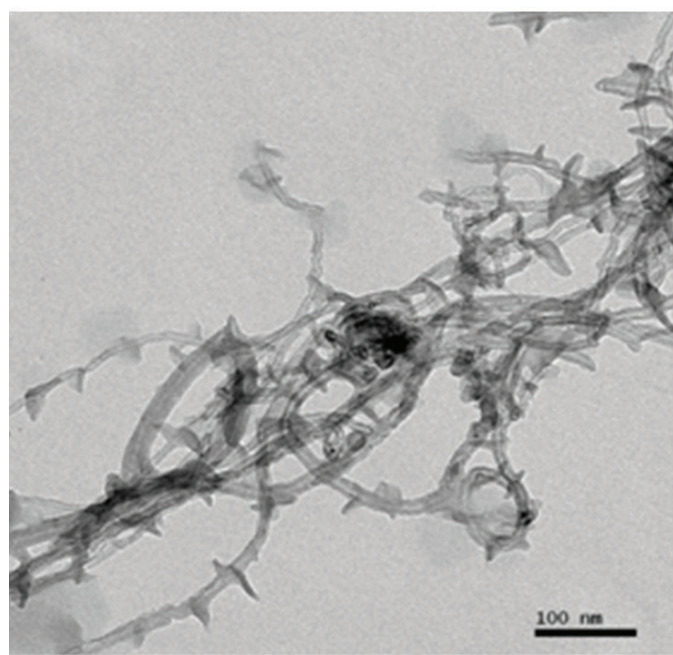

(c)

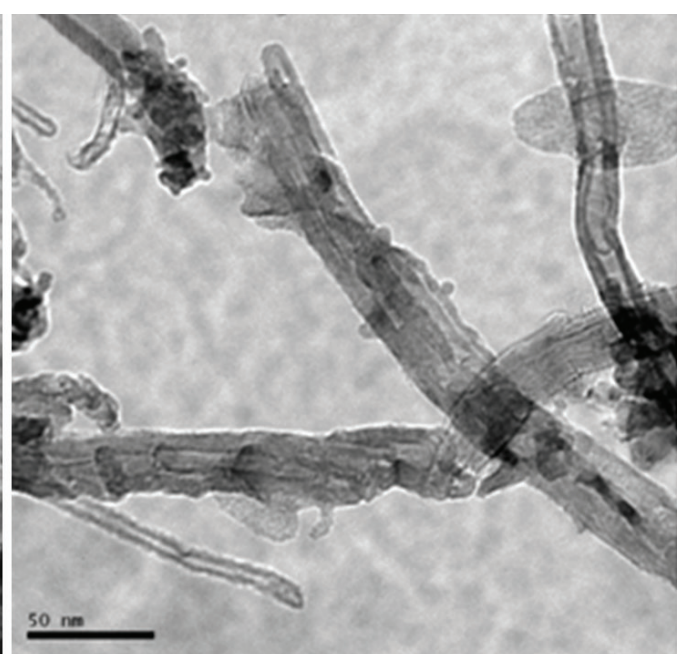

(b)

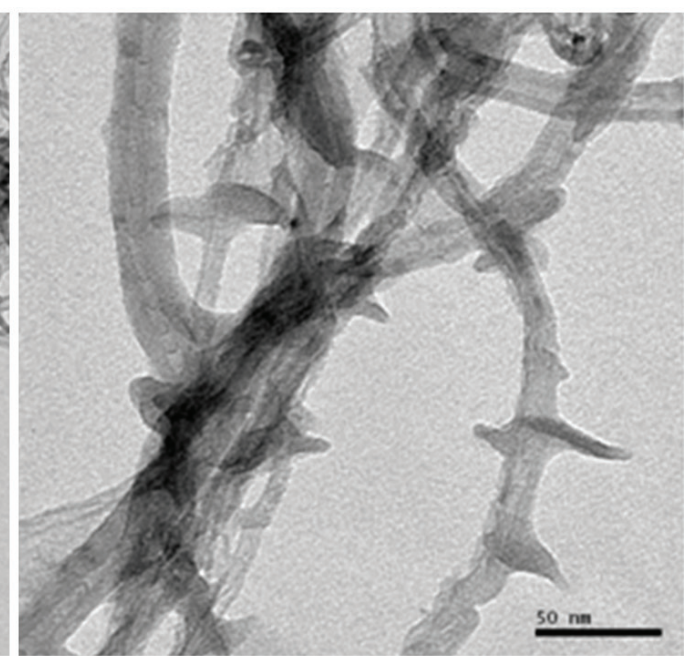

(d)

Figura 2. Micrografias de MET dos nanocompósitos: a, b - PE-N4 com 5\% (p/p) de NTC; c, d - PE-N8 com 10,5\% (p/p) de NTC. 
dos NTC puro. A partir dessas imagens pode ser visto que os NTC possuem múltiplas paredes e alguns até apresentam parede simples, cujos diâmetros variaram de 5 a 18 nm, e não se encontram aglomerados. Além disso, apresentam uma alta razão de aspecto, que é a razão entre o comprimento e a largura dos NTC.

A Figura 2 apresenta imagens de MET dos nanocompósitos com 5 e 10,5\% (p/p) de NTC. De acordo com essas imagens pode-se ver que houve mudanças na morfologia dos NTC. Na Figura 2 pode-se ver que os NTC estão bem dispersos e que o polímero cresceu enrolado nos NTC, resultando em uma nova morfologia, estrutura chamada de "shish-kebab" e que já está descrita na literatura ${ }^{[1,20]}$. Nesta morfologia os NTC estão decorados com objetos em forma de disco, que correspondem a lamelas de polietileno, resultando em uma nova morfologia nanohíbrida. Acredita-se que o crescimento epitaxial do polietileno nos NTC e o confinamento geométrico pelos NTC têm um papel na constituição dessa morfologia. Esta morfologia do revestimento pode ser facilmente controlada ajustando as condições experimentais ${ }^{[1]}$. As micrografias mostram, sem dúvidas, que o polietileno cresce sobre a superfície dos nanotubos o que ajuda a separá-los e desestruturar os agregados de NTC.

A morfologia dos nanocompósitos preparados por polimerização in situ mostra uma distribuição homogênea na matriz e um bom recobrimento do polímero na superfície do NTC.

\section{Correlação das propriedades dos nanocompósitos PE/NTC e PE/NG}

Os resultados referentes aos nanocompósitos PE/ NG foram apresentados em outros artigos ${ }^{[25,26]}$. Portanto, para que não haja repetição dos resultados e sim uma correlação entre as propriedades dos nanocompósitos com as duas nanocargas utilizadas (nanotubos de carbono e nanolâminas de grafeno), os resultados com NG serão apresentados juntamente com os de NTC. Como todos os nanocompósitos foram obtidos por polimerização in situ não é possível obter exatamente o valor de carga desejada, assim, foram selecionados conteúdos de nanocarga próximos para que o estudo seja realmente comparativo.

\section{Estabilidade térmica}

A estabilidade térmica dos nanocompósitos foi investigada por análise termogravimétrica (TGA). A Figura 3 mostra as curvas TG do polietileno puro e dos nanocompósitos de NG e NTC. Pela análise das curvas pode-se ver que quanto maior o percentual de carga maior o resíduo em cada amostra. Além disso, percentuais de NG e NTC próximos resultam em quantidade similares de resíduo. As cargas utilizadas, NG e NTC não se decompõem mesmo em altas temperaturas porque são extremamente estáveis termicamente.

Os dados provenientes das curvas TG, como temperatura inicial de degradação $\left(\mathrm{T}_{\mathrm{i}}\right)$ e temperatura em que ocorre a taxa máxima de degradação $\left(\mathrm{T}_{\max }\right)$ são apresentados na Tabela 3 .

Tanto a temperatura inicial de degradação $\left(\mathrm{T}_{\mathrm{i}}\right)$ quanto a temperatura máxima de degradação $\left(\mathrm{T}_{\max }\right)$ tiveram um aumento em ambos os nanocompósitos, quando comparadas ao polietileno puro. Entre os nanocompósitos de PE/NTC não houve diferenças significativas nas $T_{i}$ e $\mathrm{T}_{\max }$ com o aumento da nanocarga, ao contrario, os nanocompósitos de PE/NG apresentaram um aumento gradativo, principalmente na $\mathrm{T}_{\max }$. Assim, pode-se verificar que as NG foram mais eficientes, promovendo maior estabilidade térmica ao polietileno quando há maior quantidade de nanocarga. Alguns mecanismos foram sugeridos na literatura ${ }^{[3,32]}$. Os NTC dispersos podem retardar o fluxo do produto de degradação e por meio disso atrasar o começo da degradação, o mesmo ocorre com as NG. O polímero perto dos NTC pode degradar mais lentamente, o que poderia deslocar a $\mathrm{T}_{\max }$ para temperaturas mais altas. Outro mecanismo possível atribui a melhoria na estabilidade térmica ao efeito de alta condutividade térmica nos compósitos NTC/polímero que facilita a dissipação de calor dentro do compósito ${ }^{[32]}$. A melhora observada na estabilidade térmica sugere que os NTC (isto pode ser estendido às $\mathrm{NG}$ ) poderiam ser eficientes como aditivos retardantes de chama em matrizes de polímero ${ }^{[3]}$. Como as NG foram mais eficientes do que os NTC, isso sugere que as lâminas de grafeno estariam melhor dispersas e bem distribuídas na matriz polimérica do que os NTC, ou simplesmente esta diferença é um efeito da diferente morfologia das duas nanocargas.

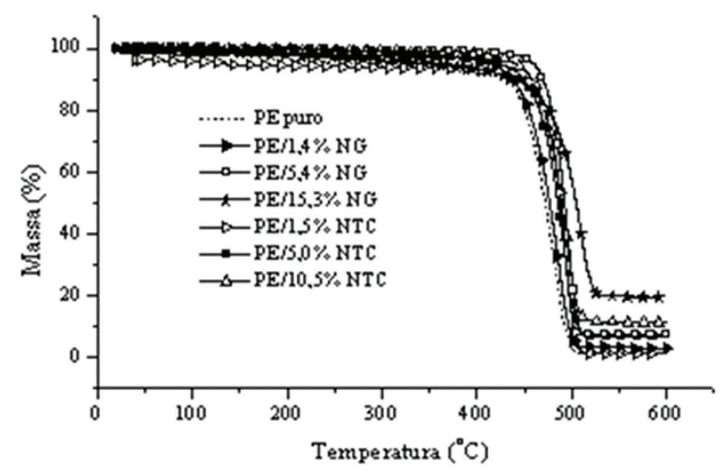

Figura 3. Curvas TG do polietileno puro e dos nanocompósitos $\mathrm{PE} / \mathrm{NG}$ e PE/NTC.

Tabela 3. Propriedades Térmicas do Polietileno Puro e dos Nanocompósitos com NTC e NG.

\begin{tabular}{|c|c|c|c|c|c|}
\hline \multirow[t]{2}{*}{ Nome da amostra } & \multicolumn{2}{|c|}{ NTC } & \multirow[t]{2}{*}{ Nome da amostra } & \multicolumn{2}{|c|}{ NG } \\
\hline & $\mathbf{T}_{i}\left({ }^{\circ} \mathbf{C}\right)$ & $\mathbf{T}_{\max }\left({ }^{\circ} \mathbf{C}\right)$ & & $\mathbf{T}_{i}\left({ }^{\circ} \mathbf{C}\right)$ & $\mathbf{T}_{\max }\left({ }^{\circ} \mathbf{C}\right)$ \\
\hline PE puro & 434 & 480 & - & - & - \\
\hline $\mathrm{PE} / 1,5 \% \mathrm{NTC}$ & 459 & 492 & $\mathrm{PE} / 1,4 \% \mathrm{NG}$ & 454 & 487 \\
\hline $\mathrm{PE} / 5,0 \% \mathrm{NTC}$ & 449 & 490 & $\mathrm{PE} / 5,4 \% \mathrm{NG}$ & 472 & 495 \\
\hline PE $/ 10,5 \%$ NTC & 452 & 492 & $\mathrm{PE} / 15,3 \% \mathrm{NG}$ & 463 & 510 \\
\hline
\end{tabular}



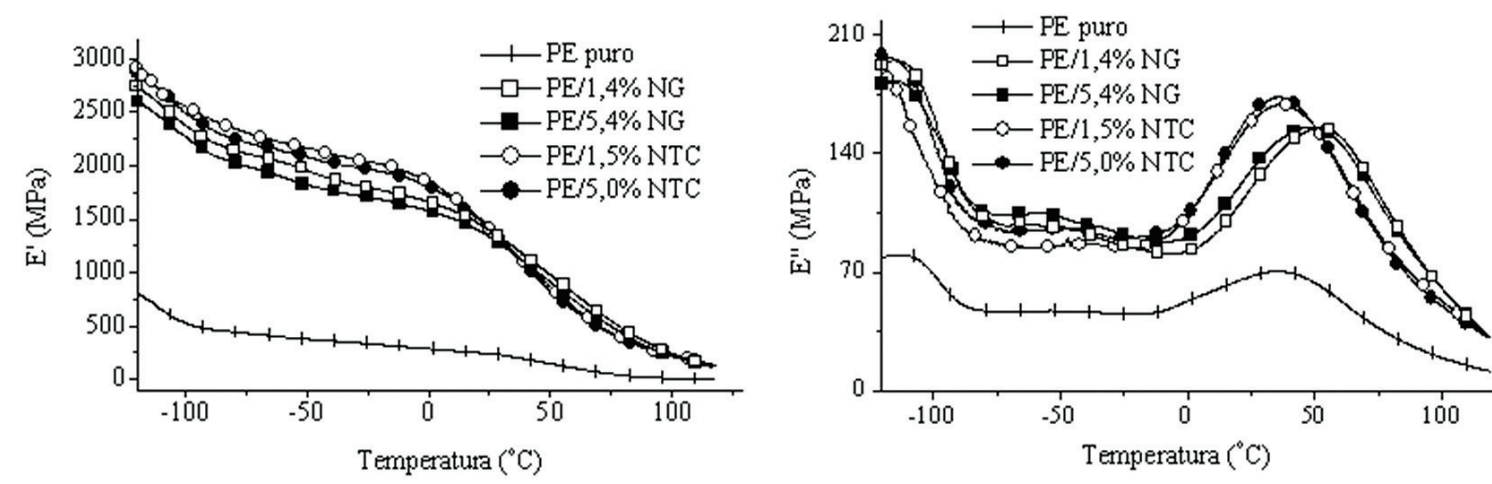

Figura 4. Variação no módulo de armazenamento (E') e no módulo de perda (E”) do polietileno puro e dos nanocompósitos em função da temperatura.

\section{Análise dinâmico-mecânica (DMA)}

As propriedades mecânicas dinâmicas dos nanocompósitos foram investigadas através da análise dinâmico-mecânica (DMA). A Figura 4 apresenta os módulos de armazenamento e de perda do polietileno puro e dos dois nanocompósitos.

De acordo com o módulo de armazenamento (E') mostrado na Figura 4, todos os nanocompósitos apresentaram valor de E' maior que o polietileno puro, sendo que acima de $100{ }^{\circ} \mathrm{C}$ as curvas apresentam uma tendência para o mesmo valor. Já entre os nanocompósitos não há uma diferença muito acentuada, sendo que os nanocompósitos de PE/NTC apresentaram valores de módulo superiores em baixas temperaturas (região de transição vítrea $-120{ }^{\circ} \mathrm{C}$ ). Mas na temperatura de trabalho, em torno de $25{ }^{\circ} \mathrm{C}$, todos os nanocompósitos apresentam o mesmo comportamento. $\mathrm{O}$ aumento no valor de E' está relacionado com aumento da rigidez da matriz polimérica. Isso quer dizer que tanto as $\mathrm{NG}$ como os NTC tornam o polietileno mais rígido.

Em relação ao módulo de perda (E”) que está relacionado com a dissipação viscosa mostrado na Figura 4, todos os nanocompósitos apresentaram valor de módulo maior que o polietileno puro e entre eles não houve uma diferença muito acentuada, do mesmo modo como ocorreu no módulo de armazenamento. $\mathrm{O}$ valor de E" maior quer dizer que há maior restrição na mobilidade das cadeias poliméricas ${ }^{[33]}$. Essa restrição na mobilidade das cadeias pode estar associada à maior rigidez da matriz de polietileno devido à presença das nanocargas.

O aumento das propriedades mecânicas dos nanocompósitos requer um alto grau de transferência de carga entre a matriz e a nanocarga. Se a adesão interfacial entre as fases é fraca, a nanocarga se comporta como buracos ou falhas, introduzindo concentrações locais de tensão, e os benefícios das propriedades dessas cargas são perdidos. Tanto as NG como os NTC precisam estar bem dispersos. No caso de má dispersão, os nanocompósitos irão enfraquecer devido à separação dos agregados da carga, resultando em uma significativa redução das propriedades mecânicas ${ }^{[34]}$. O significativo aumento do módulo elástico na Figura 4 mostra um alto grau de interação entre os nanotubos de carbono e as nanolâminas de grafeno com a matriz polimérica confirmando uma boa dispersão das nanocargas.

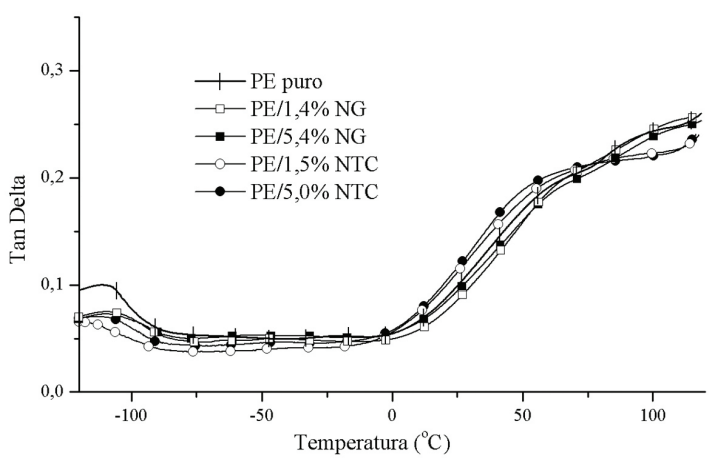

Figura 5. Variação da tan delta do polietileno puro e dos nanocompósitos com a temperatura.

A Figura 5 relaciona a variação da temperatura de transição vítrea $\left(\mathrm{T}_{\mathrm{g}}\right)$ e de tan delta do polietileno puro e dos nanocompósitos com o aumento da nanocarga. Os nanocompósitos PE/NG sofreram um leve aumento na $\mathrm{Tg}$ comparados ao polietileno puro. Este aumento não foi observado nos nanocompósitos PE/NTC. O aumento da $\mathrm{Tg}$, apesar de ser pequeno, pode significar que as NG produzem um melhor efeito de reforço no PE que os NTC (Tabela 4).

Por outro lado, a interface entre os NTC e o polietileno parece ser mais efetiva do que com as NG devido aos menores valores de tan delta (altura do pico que indica o valor de $\mathrm{T}_{\mathrm{g}}$ - região abaixo de $-100^{\circ} \mathrm{C}$ ). Este resultado corrobora com as microscopias de transmissão que mostram que o polietileno cresce ao redor dos nanotubos apresentando uma forte interação entre a nanocarga e o polímero.

A técnica de espectroscopia de impedância eletroquímica foi utilizada para investigar a condutividade dos nanocompósitos. A Tabela 5 apresenta os valores de condutividade elétrica para os nanocompósitos de PE/ NTC e PE/NG.

O polietileno puro apresenta uma condutividade elétrica de $1,4 \times 10^{-13} \mathrm{~S}_{\mathrm{cm}}{ }^{-1}$, que permanece praticamente inalterada com a adição de $1,4 \% \quad(\mathrm{p} / \mathrm{p})$ de NG $\left(2,4 \times 10^{-13} \mathrm{~S} . c m^{-1}\right)$, mas que começa a aumentar com o mesmo percentual de NTC $\left(9,8 \times 10^{-12}{\mathrm{~S} . \mathrm{cm}^{-1}}^{-1}\right.$. Uma alteração significativa da condutividade elétrica, da ordem de $10^{6}$ vezes, foi detectada com a adição de 5,0\% (p/p) de 
Tabela 4. Variação da temperatura de transição vítrea $\left(\mathrm{T}_{\mathrm{g}}\right)$ do polietileno puro e dos nanocompósitos e valores de tan delta.

\begin{tabular}{lcc}
\hline Nome da amostra & $\mathbf{T}_{\mathbf{g}}\left({ }^{\circ} \mathbf{C}\right)$ & $\begin{array}{r}\text { Tan delta } \\
(\mathbf{T}=-\mathbf{1 0 9})\end{array}$ \\
\hline PE puro & -111 & 0,100 \\
PE/1,4\%NG & -109 & 0,075 \\
PE/5,4\%NG & -109 & 0,073 \\
PE/1,5\%NTC & -114 & 0,064 \\
PE/5,0\%NTC & -110 & 0,070 \\
\hline
\end{tabular}

Tabela 5. Condutividade elétrica do polietileno puro e dos nanocompósitos PE/NG e PE/NTC.

\begin{tabular}{lc}
\hline \multicolumn{1}{c}{ Nome da amostra } & $\sigma\left(\mathbf{S . c m}^{-1}\right)$ \\
\hline PE puro & $1,4 \times 10^{-13}$ \\
PE/1,4\% NG & $2,4 \times 10^{-13}$ \\
PE/5,4\%NG & $5,5 \times 10^{-12}$ \\
PE/1,5\%NTC & $9,8 \times 10^{-12}$ \\
PE/5,0\%NTC & $1,3 \times 10^{-7}$ \\
\hline
\end{tabular}

NTC, atingindo cerca de $1,3 \times 10^{-7}{\mathrm{~S} . \mathrm{cm}^{-1}}^{\text {. Utilizando um }}$ percentual de NG similar não foi possível obter a mesma ordem de grandeza no valor da condutividade. Isso significa que os NTC estão melhores distribuídos na rede polimérica para formar um caminho condutor e com 5,0\% de NTC já é possível obter polietileno semi-condutor. Em relação às NG só foi possível obter polietileno semicondutor com $8,3 \%(\mathrm{p} / \mathrm{p})$ de nanocarga ${ }^{[26]}$.

\section{Conclusões}

Nanocompósitos de polietileno/NTC foram obtidos por polimerização in situ com boa atividade catalítica. As imagens de microscopias mostram que os NTC apresentam uma grande razão de aspecto e que o polietileno cresce ao redor dos NTC.

As duas nanocargas estudadas (NTC e NG) forneceram estabilidade térmica ao polietileno, porém a temperatura de degradação máxima foi alcançada quando se utilizou a maior quantidade de NG. Por outro lado, a temperatura de degradação não foi sensível ao aumento de NTC nos nanocompósitos indicando que as NG fornecem uma maior estabilidade térmica do que os NTC.

Os resultados de propriedades mecânicas no modo dinâmico indicam que não há diferenças significativas entre os nanocompósitos obtidos com as duas nanocargas. Enquanto os nanocompósitos PE/NTC apresentaram maior módulo de armazenamento, os nanocompósitos $\mathrm{PE} / \mathrm{NG}$ apresentaram maior Tg. O que pode ser afirmado é que os NTC tiveram maior interação com o polímero do que as $\mathrm{NG}$, provavelmente devido à morfologia da nanocarga, pois uma é formada de nanotubos e a outra de nanolâminas.

Em relação às propriedades elétricas os NTC se mostraram mais eficientes do que as NG, com 5,0\% (p/p) de NTC já se consegue obter polímero semicondutor, ao passo que com as NG é necessário 8,3\% (p/p) ${ }^{[26]}$.

Podemos concluir que as diferenças observadas entre os dois tipos de nanocompósitos podem atribuídas às suas diferenças morfológicas e não a uma diferença na dispersão das mesmas, pois o estudo das propriedades mostrou que as duas apresentam uma boa dispersão.

\section{Agradecimentos}

Os autores agradecem à CAPES, CNPq e a FAPERGS-PRONEX pelo apoio financeiro, ao Centro de Microscopia Eletrônica da UFRGS pelas analises de MET, à Nacional de Grafite Ltda pelo fornecimento de grafite Micrograf HC11 e à Bayer pelo fornecimento dos NTC - Baytubes C 150 P.

\section{Referências Bibliográficas}

1. Bredeau, S.; Peeterbroeck, S.; Bonduel, D.; Alexandre, M. \& Dubois, P. - Polym Int., 57, p.547 (2008). http://dx.doi. org/10.1002/pi.2375

2. Trujillo, M.; Arnal, M. L.; Müller, A. J.; Laredo, E.; Bredeau, S.; Bonduel, D. \& Dubois, P. - Macromolecules, 40, p.6268 (2007). http://dx.doi.org/10.1021/ma071025m

3. Moniruzzaman, M. \& Winey, K. I. - Macromolecules, 39, p.5194 (2006). http://dx.doi.org/10.1021/ma060733p

4. Wakabayashi, K.; Pierre, C.; Dikin, D. A.; Ruoff, R. S.; Ramanathan, T.; Brinson, L. C. \& Torkelson, J. M. - Macromolecules, 41, p.1905 (2008). http://dx.doi. org/10.1021/ma071687b

5. Brasil. Ministério de Minas e Energia - MME. (2009). Disponível em: <http://www.mme.gov.br/portalmme/ opencms/sgm/galerias/arquivos/plano_duo_decenal/a_ mineracao_brasileira/P28_RT41_Perfil_da_Grafita.pdf $>$.

6. Gopakumar, T. G. \& Pagé, D. J. Y. S. - Polym. Eng. Sci., 44, p.1162 (2004). http://dx.doi.org/10.1002/pen.20109

7. Novoselov, K. S.; Geim, A. K.; Morozov, S. V.; Jiang, D.; Zhang, Y.; Dubonos, S. V.; Grigorieva, I. V. \& Firsov, A. A. - Science, 306, p.666 (2004). PMid:15499015. http:// dx.doi.org/10.1126/science.1102896

8. Balandin, A. A.; Ghosh, S.; Bao, W.; Calizo, I.; Teweldebrhan, D.; Miao, F. \& Lau, C. N. - Nano Lett., 8, p.902 (2008). PMid:18284217. http://dx.doi.org/10.1021/ n10731872

9. Chung, D. D. L. - J. Mat. Sci., 37, p.1475 (2002). http:// dx.doi.org/10.1023/A:1014915307738

10. George, J. J. \& Bhowmick, A. K. - J. Mater. Sci., 43, p.702 (2008). http://dx.doi.org/10.1007/s10853-007-2193-6

11. Chen, G.; Wu, C.; Weng, W.; Wu, D. \& Yan, W. - Polymer, 44, p.1781 (2003). http://dx.doi.org/10.1016/ S0032-3861(03)00050-8

12. Debelak, B. \& Lafdi, K. - Carbon, 45, p.1727 (2007). http:// dx.doi.org/10.1016/j.carbon.2007.05.010

13. Yakovlev, A. V.; Finaenov, A. I.; Zabud'kov, S. L. \& Yakovleva, E. V. - Russ. J. Appl. Chem., 79, p.1741 (2006). http://dx.doi.org/10.1134/S1070427206110012

14. Li, J.; Liu, Q. \& Da, H. - Mater. Lett., 61, p.1832. (2007). http://dx.doi.org/10.1016/j.matlet.2006.07.142

15. Du, X. S.; Xiao, M. \& Meng, Y.Z. - Eur. Polym. J., 40, p.1489 (2004). http://dx.doi.org/10.1016/j.eurpolymj.2004.02.009

16. Kaminsky, W.; Funck, A. \& Wiemann, K. - Macromol. Symp., 239, p.1, (2006). http://dx.doi.org/10.1002/ masy. 200690084 
17. Bonduel, D.; Mainil, M.; Alexandre, M.; Monteverde, F. \& Dubois P. - Chem. Commun., 6, p.781 (2005). PMid:15685336. http://dx.doi.org/10.1039/b414164d

18. Gorrasi, G.; Bredeau, S.; Candia, C. D.; Patimo, G.; Pasquale, S. D. \& Dubois, P. - Macromol. Mater. Eng., 296, p.408 (2011). http://dx.doi.org/10.1002/mame.201000336

19. Bonduel, D.; Bredeau, S.; Alexandre, M.; Monteverde, F. \& Dubois, P. - J. Mater. Chem., 17, p.2359 (2007). http:// dx.doi.org/10.1039/b701764b

20. Bredeau, S.; Boggioni, L.; Bertini, F.; Tritto, I.; Monteverde, F.; Alexandre, M. \& Dubois, P. - Macromol. Rapid Commun., 28, p.822 (2007). http://dx.doi.org/10.1002/ marc. 200600872

21. Trujillo, M.; Arnal, M. L.; Müller, A. J.; Bredeau, S.; Bonduel, D.; Dubois, P.; Hamley, I. W. \& Castelletto, V. - Macromolecules, 41, p.2087 (2008). http://dx.doi. org/10.1021/ma702272e

22. Dubois, P. \& Alexandre, M. - Adv. Eng. Materials, 8, p.147 (2006). http://dx.doi.org/10.1002/adem.200500256

23. Li, S.; Chen, H.; Cui, D.; Li, J.; Zhang, Z.; Wang, Y. \& Tang, T. - Polym. Comp., 31, p.507 (2010).

24. Sahoo, N. G.; Rana, S.; Cho, J. W.; Li, L. \& Chan, S. H. - Prog Polym Sci., 35, p.837 (2010). http://dx.doi. org/10.1016/j.progpolymsci.2010.03.002

25. Fim, F. C.; Guterres, J. M.; Basso, N. R. S. \& Galland, G. B. - J. Polym. Sci Part A: Polym. Chem., 48, p.692 (2010). http://dx.doi.org/10.1002/pola.23822

26. Fim, F. C.; Graebin, A. P.; Basso, N. R. S.; Azambuja, D. S. \& Galland, G. B. - J. Appl. Polym. Sci., 128, p.2630 (2013). http://dx.doi.org/10.1002/app.38317
27. Montagna, L. S.; Fim, F. C.; Galland, G. B. \& Basso N. R. S. - Macromol. Symp., 299-300, p.48 (2011). http://dx.doi. org/10.1002/masy.200900133

28. Milani, M. A.; Quijada, R.; Basso, N. R. S.; Graebin, A. P. \& Galland, G. B. - J Polym Sci, Part A: Polym Chem., 50, p.3598 (2012). http://dx.doi.org/10.1002/pola.26149

29. Milani, M. A.; González, D.; Quijada, R.; Basso, N. R. S.; Cerrada, M. L.; Azambuja, D. S. \& Galland, G. B. - Compos Sci Tech., 84, p.1 (2013). http://dx.doi. org/10.1016/j.compscitech.2013.05.001

30. Potts, R.; Dreyer, D. R.; Bielawski, C. W. \& Ruoff, R. S. - Polymer, 52, p.5 (2011). http://dx.doi.org/10.1016/j. polymer.2010.11.042

31. Glotin, M. \& Mnldelkern, L. - Colloid \& Polym., 260, p.182 (1982). http://dx.doi.org/10.1007/BF01465438

32. Huxtable, S. T.; Cahill, D. G.; Shenogin, S.; Xue, L.; Ozisik, R.; Barone, P.; Usrey, M.; Strano, M. S.; Siddons, G.; Shim, M. \& Keblinski, P. - Nat. Mater., 2, p.731 (2003). PMid:14556001. http://dx.doi.org/10.1038/nmat996

33. Jayanarayanan, K.; Thomas, S. \& Joseph, K. - Compos. A, 39, p. 164 (2008). http://dx.doi.org/10.1016/j. compositesa.2007.11.008

34. Gorrasi, G.; Bredeau, S.; Candia, C. D.; Patimo, G.; Pasquale, S. D. \& Dubois, P. - Polym. Adv. Technol., 23, p.1435 (2012). http://dx.doi.org/10.1002/pat.2064

Enviado: 03/04/13

Reenviado: $23 / 12 / 13$

Aceito: $15 / 01 / 14$ 